\title{
Universal Field-Induced Charge-Density-Wave Phase Diagram: Theory versus Experiment
}

\author{
A.G. Lebed* \\ Department of Physics, University of Arizona, \\ 1118 E. 4-th Street, Tucson, AZ 85721, USA
}

\begin{abstract}
We suggest a theory of the Field-Induced Charge-Density-Wave (FICDW) phases, generated by high magnetic fields in quasi-low-dimensional conductors. We demonstrate that, in layered quasi-one-dimensional conductors, the corresponding critical magnetic fields ratios are universal and do not depend on any fitting parameter. In particular, we find that $H_{1} / H_{0}=0.73, H_{2} / H_{0}=0.59, H_{3} / H_{0}=0.49, H_{4} / H_{0}=0.42$, where $H_{n}$ is a critical field of a phase transition between the FICDW phases with numbers $n$ and $n+1$. The suggested theory is in very good qualitative and quantitative agreements with the existing experimental data in $\alpha-(\mathrm{ET})_{2} \mathrm{KHg}(\mathrm{SCN})_{4}$ material.
\end{abstract}

PACS numbers: 71.45.Lr, 74.70.Kn, 71.10.-w 
High magnetic field properties of organic conductors and superconductors have been intensively studied $[1,2]$ since a discovery of the so-called field-induced spin-density-wave (FISDW) phase diagrams [3,4]. Phase transitions from metallic to the FISDW phases were successfully explained in terms of the $3 D \rightarrow 2 D$ dimensional crossovers [5-11,1]. In particular, the metal-FISDW phase transition line was calculated in Refs. [5-7], whereas, a free energy of the FISDW phases was evaluated for all range of temperatures and magnetic fields in Refs. [8,9]. In addition, the so-called three-dimensional quantum Hall effect, experimentally observed in the FISDW phases [1-4], was theoretically explained in Refs. $[10,11]$.

A relative phenomenon - the so-called field-induced charge-density-wave (FICDW) phase diagram - was anticipated in Refs. $[5,12]$ and recently experimentally discovered in $\alpha$ $(\mathrm{ET})_{2} \mathrm{KHg}(\mathrm{SNC})_{4}$ conductor [13-18]. Although originally the FICDW phases were predicted to exist due to electron-electron interactions [12], later it was shown [19] that they naturally appeared in a physical picture, where only electon-phonon interactions were taken into account. Note that the phase diagram, suggested in Ref. [12], depends on many parameters such as details of electron-electron interactions, temperature, and anisotropy ratios of a quasi-one-dimensional (Q1D) electron spectrum. In addition, according to Ref. [12], the FICDW phases are always mixed with the FISDW ones. The above mentioned circumstances make it to be almost impossible to test the theory [12] and to compare it with the existing experiments [13-18]. In a model [19], based on electron-phonon interactions, there is no the FICDW-FISDW mixing effects, but the analysis [19] is oversimplified and, as we stress below, is not in a quantitative agreement with the experimental data.

The main goal of our Letter is to suggest a universal theory of the FICDW phase diagram, which does not depend on details of electron-electron and electron-phonon interactions as well as on temperature and details of a Q1D electron spectrum. In particular, we suggest a model, based on electron-phonon interactions, for a general form of a layered Q1D spectrum. We demonstrate that the critical magnetic fields ratios, $H_{1} / H_{0}=0.73, H_{2} / H_{0}=0.59, H_{3} / H_{0}=0.49, H_{4} / H_{0}=0.42$ (where $H_{n}$ is a critical field of a phase transition between the FICDW phases with numbers $n$ and $n+1$ ) do not depend on any parameter and calculate them. A comparison of the present theory with the experiments [14-18] shows not only qualitative but also quantitative agreement. This justifies a validity of our approach and indicates, in particular, that the electron-electron interactions and FICDW-FISDW mixing effects [12] are not very important.

Let us consider the most general layered Q1D electron spectrum, linearized near its two Fermi surface (FS) sheets,

$$
\epsilon^{ \pm}(\mathbf{p})= \pm v_{F}\left(p_{x} \mp p_{F}\right)+t_{y}^{0}\left(p_{y} a_{y}\right)+t_{z}^{0}\left(p_{z} a_{z}\right)
$$




$$
t_{y}^{0}\left(p_{y} a_{y}\right)=2 t_{y} \cos \left(p_{y} a_{y} \pm \alpha\right), \quad t_{z}^{0}\left(p_{z} a_{z}\right)=2 t_{z} \cos \left(p_{z} a_{z} \pm \beta\right)
$$

which obeys the so-called "nesting" condition $[1,2]$,

$$
\epsilon\left(\mathbf{p}+\mathbf{Q}_{\mathbf{0}}\right)+\epsilon(\mathbf{p})=0, \quad \mathbf{Q}_{\mathbf{0}}=\left[2 p_{F},(\pi-2 \alpha) / a_{y},(\pi-2 \beta) / a_{z}\right] .
$$

$\left[\right.$ Here $+(-)$ stands for right (left) sheet of Q1D FS $(1) ; p_{F}$ and $v_{F}$ are the Fermi momentum and Fermi velocity, respectively; $t_{y}$ and $t_{z}$ are overlapping integrals between electron wave functions; $p_{F} v_{F} \gg t_{y} \gg t_{z} ; \alpha$ and $\beta$ are some phase shifts; $\hbar \equiv 1$.] It is well known $[1,2,5-$ $9,12,19]$ that the so-called Peierls instability for "nested" FS (1) results in the appearance of a density wave ground state. Below, we consider a CDW ground state in accordance with the existing experimental data in $\alpha$ - $(\mathrm{ET})_{2} \mathrm{KHg}(\mathrm{SCN})_{4}$ material [13-18].

If we take into account a small (but finite) non-linearity in a Q1D electron spectrum (1) along the conducting chains, then we obtain the following electron spectrum,

$$
\begin{aligned}
& \epsilon^{ \pm}(\mathbf{p})= \pm v_{F}\left(p_{x} \mp p_{F}\right)+t_{y}\left(p_{y} a_{y}\right), \\
& t_{y}\left(p_{y} a_{y}\right)=2 t_{y} \cos \left(p_{y} a_{y} \pm \alpha\right)+2 t_{y}^{\prime} \cos \left(2 p_{y} a_{y} \pm 2 \alpha\right),
\end{aligned}
$$

with small "antinesting" term, $2 t_{y}^{\prime} \cos \left(2 p_{y} a_{y} \pm 2 \alpha\right)$, where $t_{y}^{\prime} \sim t_{y}^{2} /\left(p_{F} v_{F}\right) \ll t_{y}$. [Note that, in Eq.(3), we use a $2 \mathrm{D}$ model electron spectrum, since we suggest that $t_{y} \gg t_{z}$. In this case, the CDW and FICDW phases always correspond to an ideal "nesting" vector (2) along z-axis since the corresponding "antinesting" term is too small, $t_{z}^{\prime} \sim t_{z}^{2} /\left(p_{F} v_{F}\right) \ll t_{y}^{\prime}$.] The "antinesting" term in Eq.(3) is known to decrease a stability of the CDW ground state and, therefore, at high pressures (i.e., large enough values of $t_{y}^{\prime}$ ), metallic phase has to be restored $[1,2,5-9]$.

At first, let us discuss the FICDW phases formation, using qualitative arguments. For this purpose, we consider a Q1D electron spectrum (3) in the presence of an external magnetic field, applied along z-axis,

$$
\mathbf{H}=(0,0, H), \quad \mathbf{A}=(0, H x, 0) .
$$

To obtain electron Hamiltonian in a magnetic field (4) from the spectrum (3) we use the Peierls substitution method, $p_{x} \rightarrow-i(d / d x), p_{y} \rightarrow p_{y}-(e / c) A_{y}$, and take into account the Pauli spin splitting effects,

$$
\left[ \pm v_{F}\left(-i \frac{d}{d x} \mp p_{F}\right)+t_{y}\left(p_{y} a_{y}-\frac{\omega_{c}}{v_{F}} x\right)-\mu_{B} \sigma H\right] \Psi_{\epsilon}^{ \pm}\left(x, p_{y}, \sigma\right)=\delta \epsilon \Psi_{\epsilon}^{ \pm}\left(x, p_{y}, \sigma\right),
$$

where $\sigma=+1(-1)$ for spin up (down), $\omega_{c}=e v_{F} H a_{y} / c, \delta \epsilon=\epsilon-\epsilon_{F}$.

It is important that Eq.(5) can be solved and the corresponding wave functions can be determined analytically,

$$
\Psi_{\epsilon}^{ \pm}\left(x, p_{y}, \sigma\right)=\exp \left( \pm i p_{F} x\right) \exp \left( \pm i \frac{\delta \epsilon}{v_{F}} x\right) \exp \left( \pm i \frac{\mu_{B} \sigma H}{v_{F}} x\right) \exp \left[\mp \frac{i}{v_{F}} \int_{0}^{x} t_{y}\left(p_{y} a_{y}-\frac{\omega_{c}}{v_{F}} u\right) d u\right]
$$


Note that since $t_{y}(y)=t_{y}(y+2 \pi)$ is a periodic function of $y$ and since $\int_{0}^{2 \pi} t_{y}(y) d y=0$, then the last exponential function in Eq.(6) has to be a periodic function of $x$ with a period $2 \pi v_{F} / \omega_{c}$. Therefore, the wave functions (6) can be rewritten in a form of the Fourier series,

$$
\Psi_{\epsilon}^{ \pm}\left(x, p_{y}, \sigma\right)=\exp \left( \pm i p_{F} x\right) \exp \left( \pm i \frac{\delta \epsilon}{v_{F}} x\right) \exp \left( \pm i \frac{\mu_{B} \sigma H}{v_{F}} x\right) \sum_{n=-\infty}^{+\infty} A_{n}\left(p_{y}\right) \exp \left(i \frac{\omega_{c} n}{v_{F}} x\right)
$$

As it directly follows from Eq.(7), 2D electron spectrum (3) in a magnetic field (4), becomes pure $1 \mathrm{D}$ and corresponds to an infinite number of $1 \mathrm{D} \mathrm{FS}$, located near $p_{x} \simeq p_{F}$ and $p_{x} \simeq-p_{F}$,

$$
\delta \epsilon^{ \pm}\left(p_{x}\right)= \pm v_{F}\left(p_{x} \mp p_{F}\right)+n \omega_{c}-\mu_{B} \sigma H
$$

where $n$ is an integer quantum number. Electron spectrum (8) in shown Fig.1.

Note the a metallic phase with $1 \mathrm{D}$ spectrum (8) is unstable with respect to the CDW phases formation because of its 1D "nesting" properties. Since the FICDW instability corresponds to a pairing of an electron near $p_{F}$ and a hole near $-p_{F}$ (and vice versa) with the same spins, then we expect that possible projections along $x$-axis of the FICDW wave vectors are quantized at low enough temperatures (see Fig.1),

$$
Q_{x}^{n}=2 p_{F} \pm 2 \mu_{B} H / v_{F}+n\left(\omega_{c} / v_{F}\right), \quad \pi T \leq \omega_{c},
$$

where the quantization of the electron spectrum (8) is important. Therefore, at low temperatures, we expect a competition between the quantized FICDW order parameters (9) and have to choose the order parameter, corresponding to the highest transition temperature.

Below, we consider a problem about a formation of the FICDW phases due to electronphonon interactions by means of the Feynman diagram technique [20,21]. In particular, we consider the FICDW order parameter in the following form,

$$
\Delta(x, y)=\exp \left(i Q_{x} x\right) \exp \left(i Q_{y} y\right)+c . c ., \quad Q_{x}=2 p_{F}+q_{x}, Q_{y}=(\pi-2 \alpha) / a_{y}+q_{y}
$$

(where c.c. stands for a complex conjugated quantity), which allows to take into account deviations of the FICDW "nesting" vector from its ideal value (2) both along $x$ - and $y$-axis. In a mean field approximation, a phase transition temperature between the metallic and FICDW phases is defined by the so-called electron polarization operator [20,21],

$$
\frac{1}{g^{2}}=-\int_{0}^{2 \pi} \frac{d\left(p_{y} a_{y}\right)}{2 \pi} \sum_{\sigma} T \sum_{\omega_{n}} \int_{-\infty}^{+\infty} d x_{1} g_{--}^{\sigma}\left(i \omega_{n} ; x, x_{1} ; p_{y}-Q_{y}\right) g_{++}^{\sigma}\left(i \omega_{n} ; x_{1}, x ; p_{y}\right) \exp \left[i q_{x}\left(x-x_{1}\right)\right]
$$

where $g$ is an electron-phonon coupling constant, $\omega_{n}$ is the Matsubara frequency.

Note that Green functions of electrons near $p_{F}$ and $-p_{F}, g_{++}^{\sigma}(\ldots)$ and $g_{--}^{\sigma}(\ldots)$, respectively, can be determined from the corresponding electron wave functions (6) and spectrum 
(8) [21]. After substitution of the Green functions into Eq.(11) and some calculations, we obtain the following equations, which determine transition temperature to the FICDW phases (10),

$$
\begin{aligned}
& T_{F I C D W} \simeq \omega_{c} \exp \left[-\frac{1}{g_{\text {eff }}\left(t_{y}^{\prime}\right) g_{\text {eff }}(H)}\right], \\
& g_{\text {eff }}\left(t_{y}^{\prime}\right)=\frac{1}{2 \ln \left(t_{y}^{\prime} / t_{y}^{*}\right)}, g_{\text {eff }}(H)=M A X_{n, \delta t_{y}}\left\langle\cos \left[\phi\left(x, p_{y}\right)+n x\right]\right\rangle_{x, p_{y}}, \\
& \phi\left(x, p_{y}\right)=-\frac{4 \delta t_{y}}{\omega_{c}} \sin (x / 2) \cos \left(p_{y} a_{y}\right)+\frac{4 t_{y}^{\prime}}{\omega_{c}} \sin (x) \cos \left(2 p_{y} a_{y}\right),
\end{aligned}
$$

with the quantized $x$-component of the wave vector,

$$
q_{x}= \pm 2 \mu_{B} H / v_{F}+n\left(\omega_{c} / v_{F}\right) .
$$

[Here, $M A X_{n, \delta t_{y}}$ denotes a maximization procedure over the integer quantum number $n$ and continuous variable $\delta t_{y}$, whereas $\langle\ldots\rangle_{x, p_{y}}$ stands for an averaging procedure over the variables $x$ and $p_{y}$.] Note that a metallic phase is supposed to be stable at $H=0$, which means that $t_{y}^{\prime}>t_{y}^{*}$ in Eq.(12), where $t_{y}^{*}$ is a value of the parameter $t_{y}^{\prime}$, corresponding to a CDW phase transition at $H=0$ and $T=0$. The FICDW transition temperature (12) is calculated with the so-called logarithmic accuracy, where we use the following inequalities: $T \ll \omega_{c}$ and $t_{y}^{\prime} \ll t_{y}$.

Eq.(12) and its numerical analysis are the main results of the Letter. The distinct feature of Eq.(12) is that the ratios of the FICDW magnetic critical fields (i.e., phase transition fields to the FICDW phases with different quantum numbers (13)) do not depend on any parameter. Numerical calculations of the effective coupling constant $g_{\text {eff }}(H)$ in Eq.(12) are presented in Fig.2, where each FICDW phase is characterized by some quantum number $n$ in Eq. (13) (see the figure caption). The calculated ratios, $H_{1} / H_{0}=0.73, H_{2} / H_{0}=$ $0.59, H_{3} / H_{0}=0.49, H_{4} / H_{0}=0.42$ (where $H_{n}$ is a critical field of a phase transition between the FICDW phases with numbers $n$ and $n+1$ ) are compared with the experimental data [18] in Table 1. As it follows from the Table, there is an excellent agreement between the calculated values $H_{1} / H_{0}$ and $H_{2} / H_{0}$ and the measured ones. As to the measured ratio $H_{3} / H_{0} \simeq 0.4$, it is in a satisfactorily agreement with the corresponding calculated value, $H_{3} / H_{0}=0.49$. On the other hand, we cannot exclude [22] that, in the experiments [18], in fact, the fourth critical field, $H_{4}$, was measured instead of the third one, $H_{3}$. This would give an excellent agreement with the corresponding calculated value, $H_{4} / H_{0}=0.42$. Another important property of Eq.(12) is that the phase transition temperature is the same for two wave vectors, corresponding to signs (+) and (-) in Eq.(13).

In our opinion, a very good correspondence between the results of the present theory and the experimental data [14-18] is a strong argument in a favor of our model, based on 
TABLE I: Theoretical and experimental [18] values of the critical fields ratios for different pressures.

\begin{tabular}{ccccc}
\hline \hline Critical fields & $H_{1} / H_{0}$ & $H_{2} / H_{0}$ & $H_{3} / H_{0}$ & $H_{4} / H_{0}$ \\
\hline Theory & 0.73 & 0.59 & 0.49 & 0.42 \\
$\mathrm{P}=4 \mathrm{kbar}$ & 0.77 & 0.59 & 0.40 & - \\
$\mathrm{P}=3.5 \mathrm{kbar}$ & 0.74 & 0.57 & 0.37 & - \\
$\mathrm{P}=3 \mathrm{kbar}$ & 0.75 & 0.56 & 0.40 & - \\
\hline \hline
\end{tabular}

electron-phonon interactions. On the other hand, we point out that the previous simplified model [19] is not in a quantitative agreement with the existing experiments. Indeed, we have numerically analyzed Eq.(11) of Ref.[19] and found that, in the framework of the simplified model, $H_{1} / H_{0}=0.55, H_{2} / H_{0}=0.38$, and $H_{3} / H_{0}=0.29$, which is in obvious disagreement with the experimental data [18] (see Table 1). Therefore, it is crucial to maximize the FICDW phase transition temperature (12) over two components of the wave vector (10), $q_{x}$ and $q_{y}$, which is not done in Ref. [19]. We note that the following inequalities, $T \ll \omega_{c}$ and $t_{y}^{\prime} \ll t_{y}$, are used for the derivation of Eqs.(12),(13). Therefore, we do not take into account the finite temperature effects, described in Refs. [23,24] for the case of the FISDW phases. The next step in our studies will be to suggest a relative universal theory of the FISDW phase diagram and to compare its results with the existing experimental data. This problem will be considered in details elsewhere [25].

One of us (A.G.L.) is thankful to N.N. Bagmet (Lebed), J.S Brooks, and M.V. Kartsovnik for very useful discussions. This work was supported by the NSF grant DMR-0705986.

*Also Landau Institute for Theoretical Physics, 2 Kosygina Street, Moscow, Russia.

[1] The Physics of Organic Superconductors and Conductors, edited by A.G. Lebed (Springer, Berlin, 2008).

[2] T. Ishiguro, K. Yamaji, and G. Saito, Organic Superconductors (Springer, Berlin, 1998).

[3] P.M. Chaikin, Mu-Yong Choi, J.F. Kwak, J.S. Brooks, K.P. Martin, M.J. Naughton, E.M. Engler, and R.L. Greene, Phys. Rev. Lett. 51, 2333 (1983).

[4] M. Ribault, D. Jerome, J. Tuchendler, C. Weyl, K. Bechgaard, J. Phys. (Paris) Lett. 44, L-953 (1983).

[5] L.P. Gor'kov and A.G. Lebed, J. Phys. (Paris) Lett. 45, L-433 (1984).

[6] M. Heritier, G. Montambaux, and P. Lederer, J. Phys. (Paris) Lett. 45 L-943 (1984).

[7] G. Montambaux, M. Heritier, and P. Lederer, Phys. Rev. Lett. 552078 (1985). 
[8] A.G. Lebed, Zh. Eksp. Teor. Fiz. 89, 1034 (1985) [Sov. Phys. JETP 62, 595 (1985)].

[9] A. Virosztek, L. Cheng, and K. Maki, Phys. Rev. B 34, 3371 (1986).

[10] D. Poilblanc, G. Montambaux, M. Heritier, and P. Lederer, Phys. Rev. Lett. 58, 270 (1987).

[11] V.M. Yakovenko, Phys. Rev. B 43, 11353 (1991).

[12] D. Zanchi, A. Bjelis, and G. Montambaux, Phys. Rev. B 53, 1240 (1996); A. Bjelis, D. Zanchi, and G. Montambaux, J. Phys. IV 9, 10 (1999).

[13] J.S. Qualls, L. Balicas, J.S. Brooks, N. Harrison, L.K. Montgomery, and M. Tokumoto, Phys. Rev. B 62, 10008 (2000).

[14] D. Andres, M.V. Kartsovnik, W. Biberacher, H. Weiss, E.Balthes, H. Muller, and N. Kushch, Phys. Rev. B 64, 161104(R) (2001).

[15] D. Andres, M.V. Kartsovnik, P.D. Grigoriev, W. Biberacher, and H. Muller, Phys. Rev. B 68, 201101(R) (2003).

[16] M.V. Kartsovnik, D. Andres, W. Biberacher, C.R. Chimie 10, (2007).

[17] M.V. Kartsovnik, D. Andres, W. Biberacher, H. Muller, Physica B, 404, 357 (2009).

[18] D. Andres, M.V. Kartsovnik, W. Biberacher, K. Neumaier, I. Sheikin, and H. Muller, arXiv:0801.2696 v1 (2008).

[19] A.G. Lebed, JETP Lett. 78, 138 (2003) [Pis'ma Zh. Eksp. Teor. Fiz. 78, 170 (2003)].

[20] S.A. Brazovskii, L.P. Gor'kov, and A.G. Lebed, Zh. Eksp. Teor. Fiz. 83, 1198 (1982) [Sov. Phys. JETP 56, 683 (1982)] .

[21] A.A. Abrikosov, L.P. Gorkov, and I.E. Dzyaloshinskii, Methods of Quantum Field Theory in Statistical Physics (Dover Publications, New York, 1963).

[22] This suggestion is due to M.V. Kartsovnik (private communication).

[23] A.G. Lebed, Phys. Rev. Lett. 88, 177001 (2002).

[24] A.V. Kornilov, V.M. Pudalov, Y. Kitaoka, K. Ishida, T. Mito, J.S. Brooks, J.S. Qualls, J.A.A.J. Perenbom, N. Tateiwa, and T.C. Kobayashi, Phys. Rev. B 65, 060404R (2002).

[25] A.G. Lebed, in preparation. 


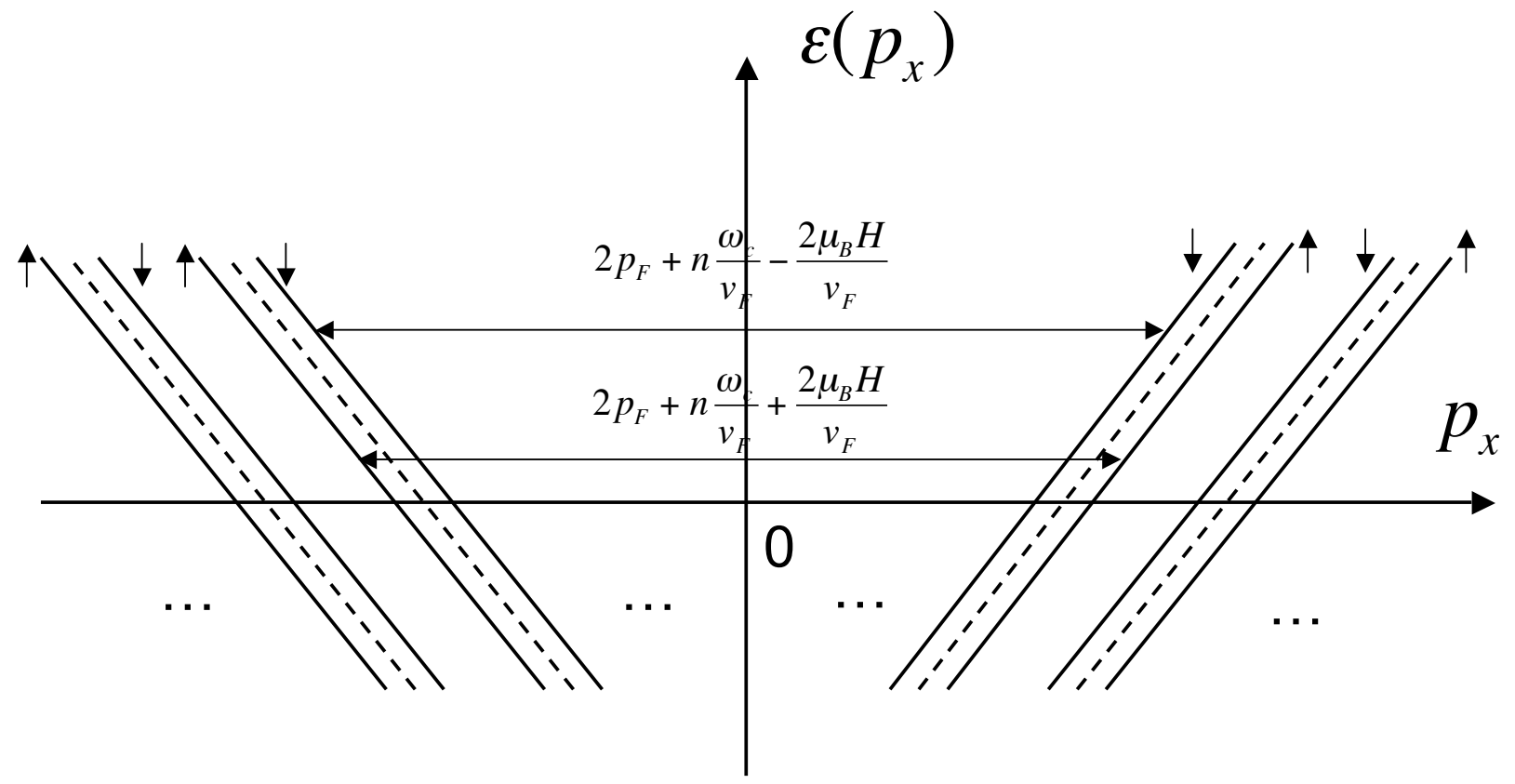

FIG. 1: A schematic view of the quantized electron spectrum (8) near $p_{x} \simeq p_{F}$ and $p_{x} \simeq-p_{F}$. There exist an infinite number of 1D Fermi surfaces, characterized by quantum number $n$, with each of them being split due to an electron spin. As a result, at low enough temperatures, there exist a competition between infinite number of "nesting" vectors, corresponding to Eq.(9). 


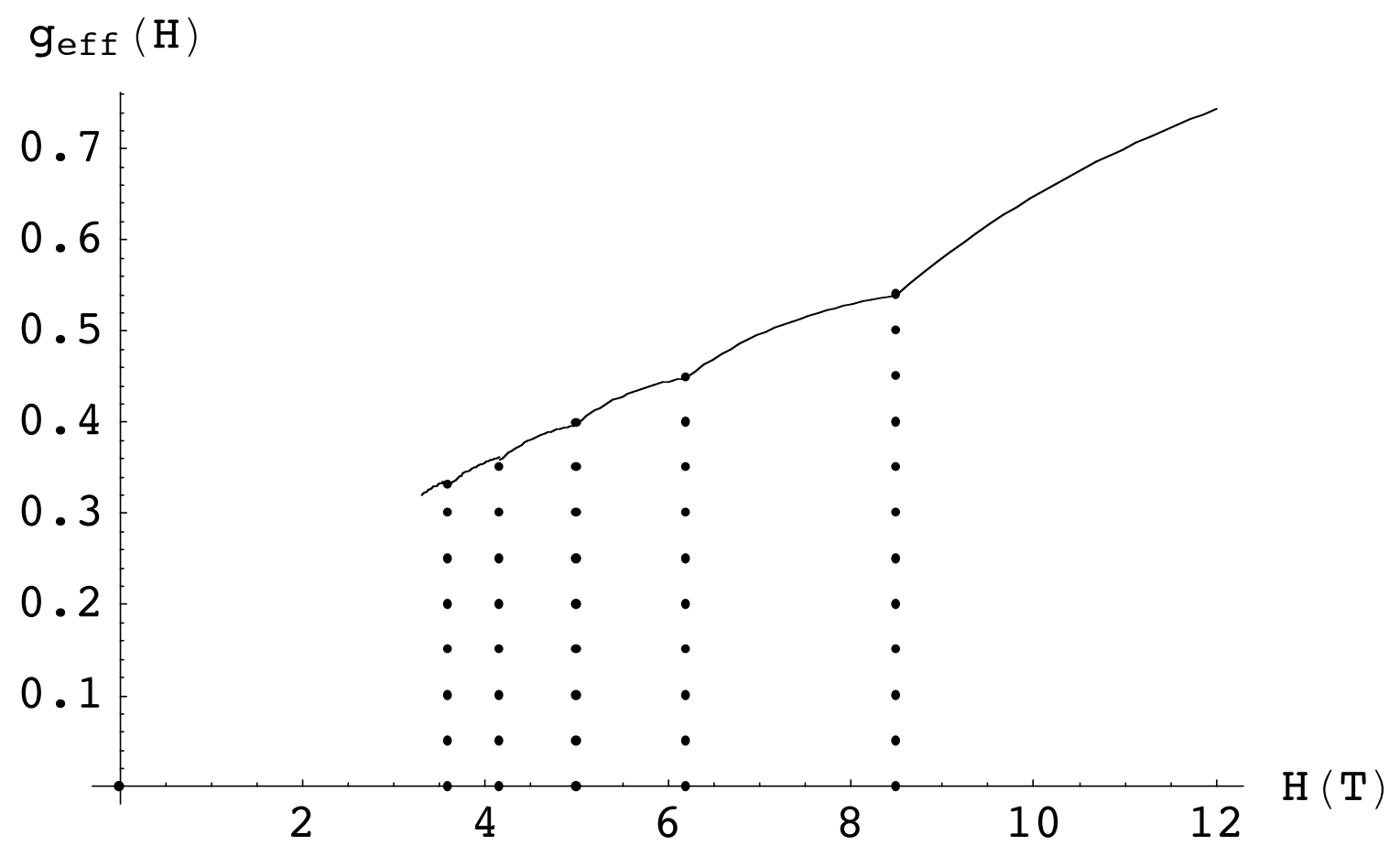

FIG. 2: Numerically calculated effective coupling constant, $g_{\text {eff }}(H)$, which defines the metalFICDW phases transition temperature (see Eq.(12)), is shown by a solid line. Phase transitions between different FICDW phases, characterized by different quantum numbers $n$ in Eq.(13), are shown by dotted lines. Phase $n=0$ corresponds to $H>8.5 T$, phase $n=1-8.5 T>H>6.2 T$, phase $n=2-6.2 T>H>5 T$, phase $n=3-5 T>H>4.15 T$, phase $n=4-4.15 T>H>3.6 T$, phase $n=5-3.6 T>H$. 